(

updates

Cite as

Nano-Micro Lett.

(2020) $12: 37$

Received: 24 October 2019

Accepted: 25 December 2019

Published online: 24 January 2020

(C) The Author(s) 2020

\title{
Hydroiodic Acid Additive Enhanced the Performance and Stability of PbS-QDs Solar Cells via Suppressing Hydroxyl Ligand
}

Xiaokun Yang ${ }^{1,2,3}$, Ji Yang ${ }^{1}$, Jahangeer Khan $^{1}$, Hui Deng ${ }^{1,3}$, Shengjie Yuan ${ }^{1,3}$, Jian Zhang ${ }^{1}$, Yong Xia ${ }^{4}$, Feng Deng ${ }^{5}$, Xue Zhou ${ }^{5}$, Farooq Umar ${ }^{1}$, Zhixin Jin ${ }^{1,2}$, Haisheng Song ${ }^{1,3 凶}$, Chun Cheng ${ }^{2}$, Mohamed Sabry ${ }^{6,7}$, Jiang Tang ${ }^{1,3}$

Xiaokun Yang and Ji Yang have contributed equally to this work.

$\triangle$ Haisheng Song, songhs-wnlo@mail.hust.edu.cn; Chun Cheng, chengc@ sustc.edu.cn

1 Wuhan National Laboratory for Optoelectronics, Huazhong University of Science and Technology, Luoyu Road 1037, Wuhan 430074, People's Republic of China

2 Department of Materials Science and Engineering and Shenzhen Key Laboratory of Nanoimprint Technology, South University of Science and Technology, Shenzhen 518055, People's Republic of China

3 Shenzhen R\&D Center of Huazhong University of Science and Technology, Shenzhen 518000, People's Republic of China

4 School of Optical and Electronic Information, Huazhong University of Science and Technology, 1037 Luoyu Road, Wuhan 430074, People's Republic of China

5 National Center for Magnetic Resonance in Wuhan, State Key Laboratory of Magnetic Resonance and Atomic and Molecular Physics, Wuhan Institute of Physics and Mathematics, Chinese Academy of Sciences, Wuhan 430071, People's Republic of China

6 Physics Department, College of Applied Science, Umm Al-Qura University, Mecca, Kingdom of Saudi Arabia

Solar Physics Lab, National Research Institute of Astronomy and Geophysics, Cairo, Egypt

\section{HIGHLIGHTS}

- The hydroiodic acid was explored systematically to modify PbS quantum dots (QDs) ink process, which could remove trap states by hydroxyl ligand and improve iodine passivation on the PbS-QDs surface.

- This strategy solved the essential question of PbS-QDs ink process and showed the favorable application prospects in QDs technology.
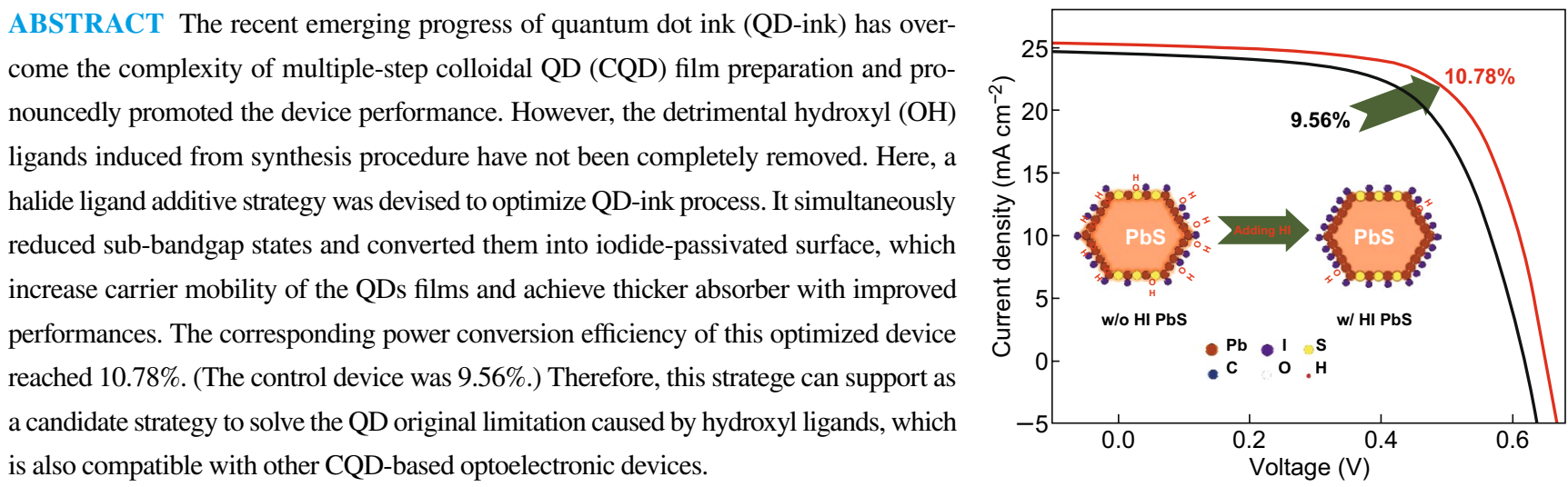

KEYWORDS Hydroxyl ligand; HI additive; Surface passivation; Quantum dots ink; Solar cells 


\section{Introduction}

Solution-processed $\mathrm{PbS}$ colloidal quantum dots (CQDs) are among the emerging materials for third-generation photovoltaics in view of their simple process [1], large scale [2], lowcost manufacturing [3], and size-dependent bandgaps [4, 5]. In the past decade, surface passivation [6-8] and device architecture [9-14] have been implemented to improve the photovoltaic performances; the efficiencies of $\mathrm{PbS}$ QD solar cells have been realized continuous breakthroughs. Recently, QDs-ink process as a new effective technique applied in PbS-QDs solar cells to refresh a new record of power conversion efficiency (PCE) of 12.6\% [15]. Therefore, it is a key to further optimize above QDs-ink [15-17] process so as to promote solar cells performance and its industrialization.

In typical CQD synthesis, the QD surface was capped by long-chain oleate surfactants in the representive (001) and (111) facets for passivation and stabilization $[6,18,19]$. Recently, Zherebetskyy et al. [18] reported hydroxyl as a parasitic surface ligand also participated in PbS-QDs surface and played a key role in stabilizing the $\mathrm{PbS}$ (111) facet. That is, the synthesized QDs have two well-defined (001) and (111) facets, and the nonpolar (001) facet can be covered by oleate to keep stable state. However, the entire polar (111) facets cannot be fully bonded by the steric hindrance of $\mathrm{OA}^{-}$molecules instead of demanding a smaller hydroxyl ligand $(\mathrm{OH})$ to preserve QDs (Fig. 1a) overall charge neutrality and minimize surface energy [18]. Unfortunately, hydroxyl groups have been proved to introduce sub-bandgap states leading to charge recombination in PbS-QDs solar cells $[16,20,21]$, which was detrimental for device performance and photostability. To solve this problem, a series of methods have been developed to eliminate the hydroxyl such as QDsink process [16, 17], synthesis precursor selection [22], and thermal annealing [21]. The world record efficiency (12.6\%) was achieved from the QD-ink process strategy [15]. However, the hydroxyl as an inherent defect cannot be removed $[16,21]$ completely in QDs-ink process. Thus, it remains an open challenge for this technology to overcome the hydroxyl effect.

It is well known that the short slab of PbS-QDs for photovoltaic technology is the imperfect surface passivation, which has a remarkable impact on their energy bands $[11,23]$, trap states [24], carrier diffusion length [8, 13, 25], and stability $[3,21]$. We explore a method to remove hydroxyl ligand from PbS-QDs surface and introduce a reliable ligand ion binding to the QD surface for further passivation. Then, we attempt to use one halogenic acid that can be deprotonated to react with hydroxyl ligands and enable halide ions to bind the $\mathrm{Pb}$ terminal facet. As the most stable halide ligand for PbS-QDs is iodine ion $[2,11]$, we perform a suitable amount of mild $\mathrm{HI}$ additive in $\mathrm{PbI}_{2}-\mathrm{PbS} \mathrm{DMF}$ solution for above motivation.

According to above scenario, here we report a facile HI additive in PbS-QD-ink system to facilitate the control of ligand reactivity and improve the QD-ink stability. It can favor the detachment of hydroxyl groups from (111) facets and promote iodide ligands binding to $\mathrm{Pb}$ atoms. (a)

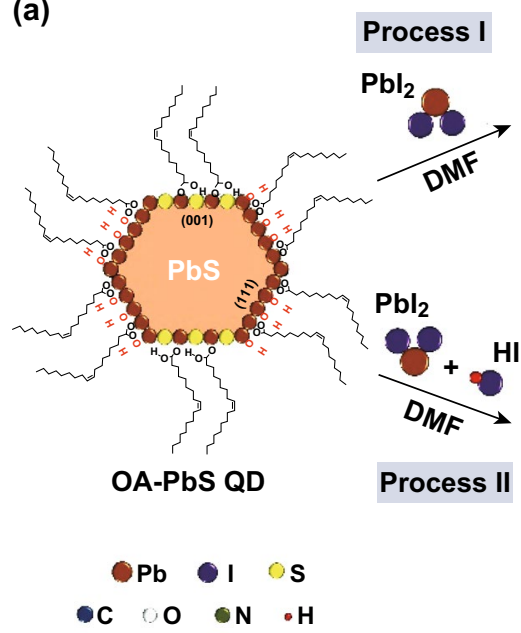

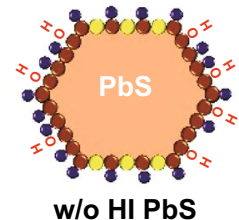

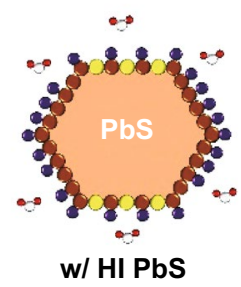

(b)
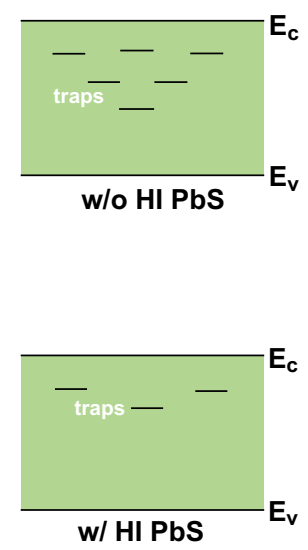

Fig. 1 a A schematic description of the solution-ligand exchange of $\mathrm{PbI}_{2}$ treated $\mathrm{PbS}-\mathrm{QD}$ (Process I) and $\mathrm{HI}-\mathrm{PbI}{ }_{2}$ treated $\mathrm{PbS}-\mathrm{QDs}(\mathrm{Process}$ II). b Corresponding trap level evolution of $\mathrm{PbS}-\mathrm{QDs}$ treated by $\mathrm{PbI}_{2}$ and $\mathrm{HI}-\mathrm{PbI}_{2}$ ligands 
The PbS-QDs film treated by the new $\mathrm{HI}-\mathrm{PbI}_{2}$ ligands has obtained a longer carrier diffusion length and lower trap density. This optimized PbS-QDs solar cells have obtained PCE of $10.78 \%$ and exhibited superior operation stability comparing with control devices. The developed ligand additive engineering strategy pronouncedly suppresses the original detrimental effects of hydroxyl and acts as effective strategy to promote the progress of QD technologies $[26,27]$.

\section{Materials and Method}

\subsection{Materials}

$\mathrm{Zn}\left(\mathrm{CH}_{3} \mathrm{COO}\right)_{2} \cdot 2 \mathrm{H}_{2} \mathrm{O}$ (Sinopharm, $\geq 99 \%$ ), monoethanolamine (Sinopharm, 99\%), Ethanedithiol (Sinopharm, $\geq 99 \%$ ), hydroiodic acid (Sinopharm, 45\%), lead oxide $\mathrm{PbO}$ (Alfa, 99.9\%), oleic acid (OA) (Alfa Aesar, 90\%), 1-octadecene (ODE) (Aladdin, $\geq 90 \%$ ), hexamethyldisilathiane (TMS) (Tci, 95\%), octane (Sinopharm, $\geq 95 \%$ ), acetone (Sinopharm, $\geq 99.5 \%$ ), ethanol (Sinopharm, $\geq 99.7 \%$ ), isopropyl alcohol (Sinopharm, 99.7\%), 1,2-ethanedithiol (EDT) (Aladdin, 97\%), acetonitrile (Sinopharm, $\geq 99.8 \%$ ), lead iodide ( $\left.\mathrm{PbI}_{2}\right)$ (Aldrich, 99\%), dimethylformamide (DMF) (Aladdin, 99.8\%), butylamine (BTA) (Aladdin, 98\%), 1-ethyl-3-methylimidazolium iodide (EMII) (Alfa, 97\%), tetramethylammonium hydroxide pentahydrate (TMAH) (Aladdin, 97\%).

\subsection{Fabrication and Characterization}

\subsubsection{Preparation of ZnO Film by Sol-Gel Method}

The $\mathrm{ZnO}$ precursor was spin-coated on ITO glass at $4000 \mathrm{r} \mathrm{min}^{-1}$ for $30 \mathrm{~s}$ under ambient environment and then annealed at $320^{\circ} \mathrm{C}$ for $12 \mathrm{~min}$.

\subsubsection{PbS CQDs Synthesis}

Oleate-capped PbS CQDs were synthesized under Schlenkline conditions according to previous reports [2] with slight modifications. A mixture of lead oxide, oleic acid, and 1-octadecenein a flask was degassed and heated for $12 \mathrm{~h}$. After that, TMS was injected into lead oleate solution under vigorous stirring. After purification, the final separated QDs were re-dispersed in octane with a $30 \mathrm{mg} \mathrm{mL}^{-1}$ for solar cell fabrication.

\subsubsection{Device Fabrication}

PbS-CQD films fabricated by solution-phase ligandexchange process serves as the main light-absorbing layer; the oleic acid-capped CQDs (OA-CQDs) could be changed into halide-passivated CQDs under air as described in previous reports [16]. Halide ligand was prepared by $\mathrm{PbI}_{2}-\mathrm{DMF}$ solution for ligand exchange. The OA-CQDs in octane were mixed with the as-prepared DMF solution. The ligand exchanged PbS-CQDs were dried to get CQD powder. The obtained iodide-passivated PbS CQDs were re-dispersed in mixed solvent butylamine (BTA) and $N, N$-dimethylformamide (DMF) with desired concentrations for absorber deposition. For modified QDs-ink process, it is similar to above process and the only difference is the amount of $\mathrm{HI}$ additive in $\mathrm{PbI}_{2}$ ligand solution. After that, two PbS-EDT layers as an hole extraction layer were fabricated via a layer-by-layer method. Finally, $\sim 80 \mathrm{~nm}$ Au was deposited by thermal evaporation at low pressure $\left(<4 \times 10^{-3} \mathrm{~Pa}\right)$.

The exact details were shown in supporting information.

\section{Results and Discussion}

A schematic diagram of the solution-phase ligand-exchange process is present in Fig. 1a. Before the ligand exchange, PbS-CQDs are capped with $\mathrm{OA}^{-}$and $\mathrm{OH}^{-}$groups. During the $\mathrm{PbI}_{2}$ ligand exchange (Process I), the long-chain and bulky oleate ligands are partially replaced by $\mathrm{I}^{-}$anions, but some hydroxyls hold strong bond with $\mathrm{Pb}$ atom of $\mathrm{PbS}-(111)$ terminate facet, leading to detrimental effect to device performance [16, 21, 22]. After adding some amount of $\mathrm{HI}$ additive in the $\mathrm{PbI}_{2}$ ligand exchange (Process II), not only the OA groups are exchanged by iodine ligands, but also the hydroxyls are expected to be eliminated by $\mathrm{HI}$ to form a small quantity of free water. For such modified ligand exchange, the trap states are significantly purified comparing with the former one (Fig. 1b). Here, we denoted the HI additive in $\mathrm{PbI}_{2}-\mathrm{PbS}-\mathrm{QDs}$ process as $\mathrm{w} / \mathrm{HI}$, without $\mathrm{HI}$ additive devices as w/o HI or control ones, and oleic acid-capped $\mathrm{PbS}-\mathrm{QDs}$ as $\mathrm{OA}-\mathrm{PbS}$.

To confirm our strategy effect, we measured Fourier-transform infrared (FT-IR) for QD films w/o and w/HI additive to 
explore the evolution of surface organic groups. The similar weak signal of oleate and hydroxyl ligands is probed in w/o and w/HI-PbS compared to OA-PbS-QDs (Fig. S2a) $\left(\mathrm{C}-\mathrm{H}_{\mathrm{x}}\right.$ vibrations at $2852-2922$ and $1380-1460 \mathrm{~cm}^{-1}$, $\mathrm{COO}^{-}$vibrations at $1400-1545 \mathrm{~cm}^{-1}$, $\mathrm{OH}^{-}$vibrations at $3200-3600 \mathrm{~cm}^{-1}$ ) [2, 16], revealing most of OA and hydroxyl ligands were removed by QDs-ink process, only some of residual signal could be detected. For detailed comparison, the $\mathrm{OH}^{-}$vibration [28] peaks of QDs-ink films are highlighted by red square (Fig. S2b). After we added HI in the ligand solution, the $\mathrm{OH}^{-}$peak intensities were reduced two times and the left oleate group signal was also removed. Those results are roughly consistent with our hypothesis, which expect that the HI additive could help to eliminate $\mathrm{OH}^{-}$. We further studied the terminal functional groups using ${ }^{1} \mathrm{H}$ nuclear magnetic resonance (NMR) spectroscopy. The comparisons among the spectra of background signals (Fig. S3) and ligand exchanged QDs (Fig. S4) indicated that a small amount of oleate residue existed in ink-processed $\mathrm{PbS}-\mathrm{QD}$. After $\mathrm{HI}$ was added in $\mathrm{PbI}_{2}-\mathrm{PbS}$, the hydroxyl peak at 4.48 ppm was suppressed and a broader $\mathrm{H}_{2} \mathrm{O}$ signal at 3.82-4.0 ppm appeared. It further proved that the HI additive could react with $\mathrm{OH}$ group by deprotonation reaction to form the free $\mathrm{H}_{2} \mathrm{O}$.

To enrich the evidence of hydroxyl groups in PbS-QDs, we also employed solid-state ${ }^{1} \mathrm{H}$ NMR to detect the hydroxyl from the w/and w/o HI-PbS-QDs powders, which is more close to work condition of these films without any solvents affection. The $\mathrm{Pb}(\mathrm{OH})_{2}$ powders were used as reference sample. The w/o HI-PbS + TMAH was obtained by adding $2 \%$ mole ratio (related to $\mathrm{PbI}_{2}$ ) TMAH into w/o HI-PbS and was checked by solid-state NMR. As shown in Fig. 2a, b, the w/HI-PbS shows lower intensity of hydroxyl groups than w/o HI-PbS powders. It means that HI additive can suppress hydroxyl in PbS-QDs, which is consistent with IR results. To explore the role of hydroxyl group in these samples, we measured the photoluminescence quantum yield (PLQY) of these powders in solution (Fig. 2c). The PLQY of w/o HI-PbS is $13.79 \%$, w/HI-PbS is $17.94 \%$, while the w/o HI-PbS + TMAH shows lowest PLQY (5.17\%), which indicates much hydroxyl along with sub-bandgap states or trap states was suppressed in w/HI-PbS-QDs. Based on above results, we can conclude that the hydroxyls reside in ligand exchanged PbS-QDs ink and introduces trap states in PbS-QDs. Using suitable amount $\mathrm{HI}$ as additive in PbS$\mathrm{PbI}_{2}$-DMF system, it can play a role of suppression to the its related trap states.

In order to optimize the content of $\mathrm{HI}$ additive in the PbSQDs, a various amount of $\mathrm{HI}$ additive was utilized in $\mathrm{PbI}_{2}$ ligand solution. As shown in absorption spectra (Fig. S5a, b), there is no appreciable change in exciton peak between w/o HI and w/HI films with low concentration of HI addition (within 5\%). However, more HI additive incorporated in $\mathrm{PbI}_{2}-\mathrm{PbS}-\mathrm{QDs}$ solution, the monodispersity of QDs (Fig. $\mathrm{S} 5 \mathrm{c}, \mathrm{d})$ degraded because of fusion or decomposition in PbSQDs. Such results also confirmed by X-ray diffraction and PL spectra (Fig. S6). Therefore, it is necessary using small amount of $\mathrm{HI}$ as additive in PbS-QDs ink to preserve the native properties of PbS-QDs without structure degradation.

To discuss the changes of the hydroxyl ligands of w/o and w/HI treated $\mathrm{PbS}$-QDs, we performed X-ray photoelectron spectroscopy (XPS) to investigate the species in QD films. As shown in Figs. 3a and S7, the O $1 s$ peaks are normalized by $\mathrm{Pb}$ peak area, and $\mathrm{w} / \mathrm{HI}$ films obtain lowest intensity of $\mathrm{O} 1 s$,
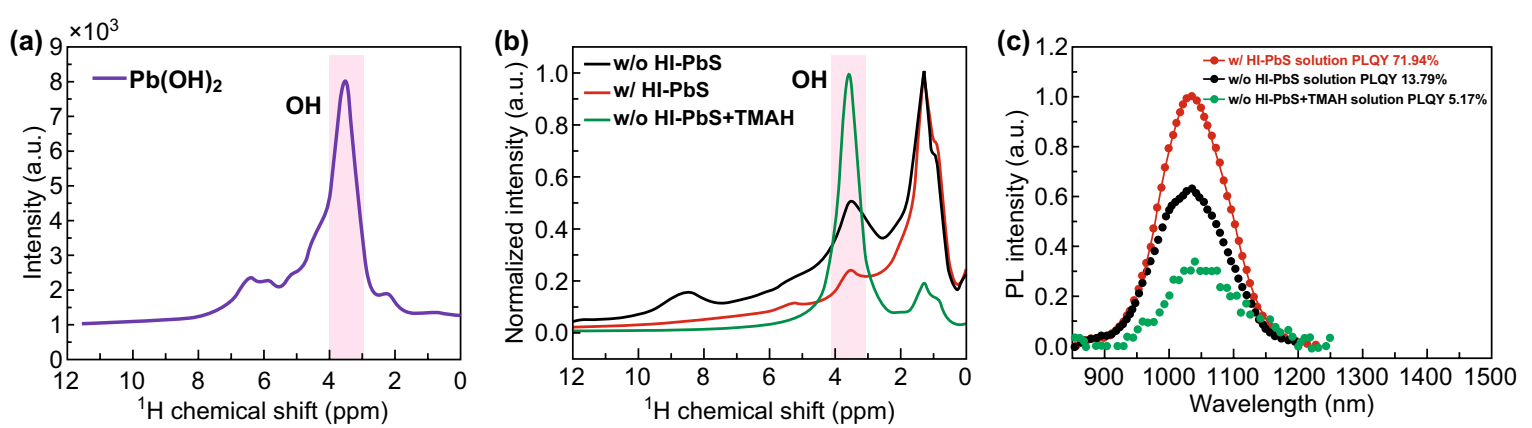

Fig. 2 a ${ }^{1} \mathrm{H}$ NMR for $\mathrm{Pb}(\mathrm{OH})_{2}$ powders, ${ }^{1} \mathrm{H}$ NMR for w/o, w/HI-PbS, and HI-PbS + TMAH powders. c Relative PL intensity of w/o, w/HI$\mathrm{PbS}$, and $\mathrm{HI}-\mathrm{PbS}+\mathrm{TMAH}$ QDs in solutions. The only $\mathrm{H}$ signal for $\mathrm{Pb}(\mathrm{OH})_{2}$ was from hydroxyl group, which was located at $\sim 3.6 \mathrm{ppm}$ 
revealing the minimum residual $\mathrm{OA}^{-}$ligand compare to others. The $\mathrm{O} 1 s$ peak can be deconvolved into $\mathrm{Pb}-\mathrm{O}(529.6 \mathrm{eV})$, $\mathrm{Pb}-\mathrm{OH}(531.3 \mathrm{eV})$, and $\mathrm{COO}^{-}(532.0 \mathrm{eV})[16,21]$. For OA$\mathrm{PbS}$ film, the $\mathrm{O} 1 s$ peak is dominated by hydroxyl and oleate ligand (Fig.S7b), which are introduced from synthesis process to bind PbS-QDs surfaces [18]. As shown in Fig. 3a-c and Table 1 , the $\mathrm{Pb}-\mathrm{OH}$ peak intensity of $\mathrm{w} / \mathrm{HI}$ film decreases near two times comparing with w/o HI film, which indicated that hydroxyl had been suppressed by adding $\mathrm{HI}$ additive in the ligand solution. It is worth noting that the $\mathrm{COO}^{-}$group has the lowest signal comparing with other two sub-peaks of O $1 s$ (Fig. 3b, c); Zherebetskyy et al. attributed this result to the $\mathrm{OA}^{-}$ligands, which has higher possibility for inelastically scattering than $\mathrm{OH}^{-}$ligand, resulting in an underestimate of the actual relative amount of $\mathrm{COO}^{-}$and $\mathrm{OH}^{-}$groups [18]. I $3 d$ peaks are also normalized by $\mathrm{Pb}$ peak area as shown in Fig. 3d. It is clear that $\mathrm{w} / \mathrm{HI}$ films hold higher I $3 d$ intensity than w/o HI films. From Fig. 3d-f, the Pb-I peak [21, 29, 30] can be deconvolved into three components. From high to low energy, those binding energies for I $3 d_{2 / 5}$ are $620.2,619.5$, and $618.4 \mathrm{eV}$, respectively. (All are constrained to FWHM of $<1.1 \mathrm{eV}$ and within $<0.2 \mathrm{eV}$ deviation.) The higher energy $(620.2 \mathrm{eV})$ component corresponds to iodide ions loosely binding to organic cations or weakly attaching on the QD surface; others lower energy component belongs to iodide binding to $\mathrm{Pb}$ atoms [21]. The results of w/HI film show a decrease of the higher energy peaks and a corresponding increase in the lower energy peaks. By calculating the integral area of I
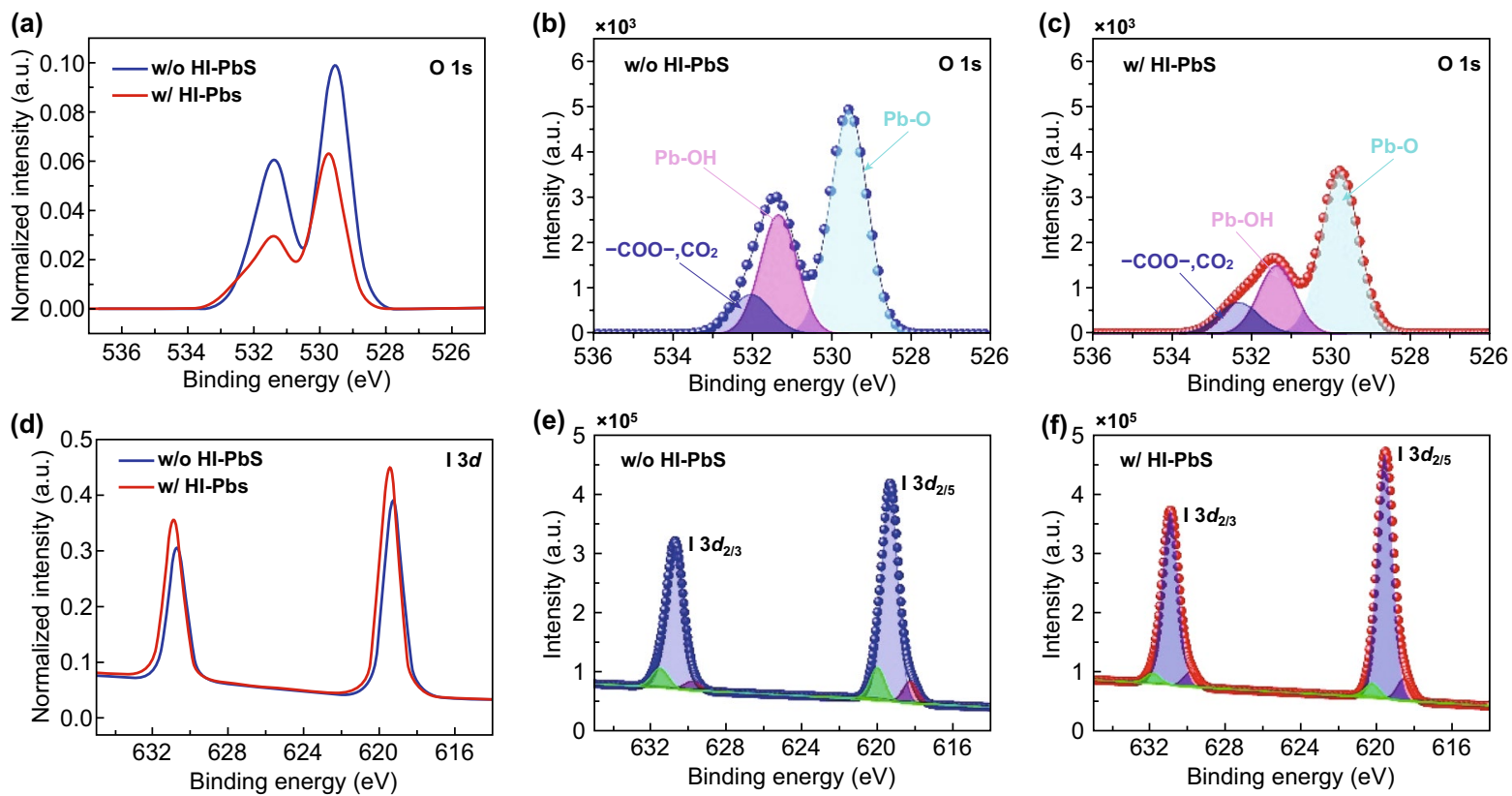

Fig. 3 XPS spectra of w/and w/o HI-PbS-QDs. a Signal from O $1 s$ of two types QDs-ink samples. b Signal of O $1 s$ from the w/o HI films. c Signal of O $1 s$ from w/HI films. d Signal from I $3 d$ of two types QDs-ink samples. e Signal of I $3 d$ from the w/o HI films. f Signal of I $3 d$ from w/HI films. The total peak areas of O $1 s$ and I $3 d$ spectra are normalized to the Pb peak area of the two type samples in a and $\mathbf{d}$, respectively. To compare the relative values, the real intensity of respective peaks from $\mathbf{b}, \mathbf{c}, \mathbf{e}$, and $\mathbf{f}$ are subtracted with background signal

Table 1 Fitting parameters and quantitative analysis of $\mathrm{O} 1 s$ spectra of w/o and w/HI PbS-QDs films in Fig. 3

\begin{tabular}{llllll}
\hline PbS-QDs & Component & Peak & FWHM & Area (\%) & Component ratio \\
\hline w/o HI PbS-QDs & $\mathrm{Pb}-\mathrm{O}$ & 529.6 & 1.1 & 55.7 & 0.12 \\
& $\mathbf{P b}-\mathbf{O H}$ & 531.3 & 1.2 & 33.6 & $\mathbf{0 . 0 7 1}$ \\
& $\mathrm{COO}, \mathrm{CO}_{2}$ & 532.0 & 1.2 & 10.7 & 0.022 \\
w/HI PbS-QDs & $\mathrm{Pb}-\mathrm{O}$ & 529.7 & 1.1 & 59.7 & 0.084 \\
& $\mathbf{P b}-\mathbf{O H}$ & 531.4 & 1.2 & 28.0 & $\mathbf{0 . 0 3 2}$ \\
& $\mathrm{COO}, \mathrm{CO}_{2}$ & 532.3 & 1.2 & 12.3 & 0.017 \\
\hline
\end{tabular}

The bold words were marked to highlight the changes of trap groups, OH group in QDs, in the XPS results 
$3 d$ peak, it can be found the $\mathrm{I} / \mathrm{Pb}$ ratio increases from $0.65: 1$ to $0.76: 1$ (Fig. S8). Based on the O $1 s$ and I $3 d$ signal changes in w/o and w/HI films, we consider that this effect is attributed to the reduced $\mathrm{OH}$ groups and an increase in iodide atoms that are strongly bound to the surface of the QDs. In view of the above results (FT-IR, NMR, and XPS), we can conclude that $\mathrm{HI}$ acting as additive reacts with hydroxyl ligand via a deprotonation reaction and enables iodide to bind on PbS-QDs surface with enhanced passivation. It should be noted a similar work [31] has implemented in big size QDs-based infrared solar cells. They focused on the removal of original ligand and improvement in CQD packing; there was limited elaboration for the content changes and evolution between hydroxyl and iodine. Our work paid more attention to the suppression of hydroxyl and passivation of iodine ligand, therefore, made this evolution process more clear.

To identify the effects of $\mathrm{HI}$ additive, we systematically optimized the amount of $\mathrm{HI}$ additive in $\mathrm{PbI}_{2}$-DMF ligand solution (Fig. 4) and the thickness of absorber for both w/ and w/o HI devices (Fig. S9). In w/HI devices, the optimized

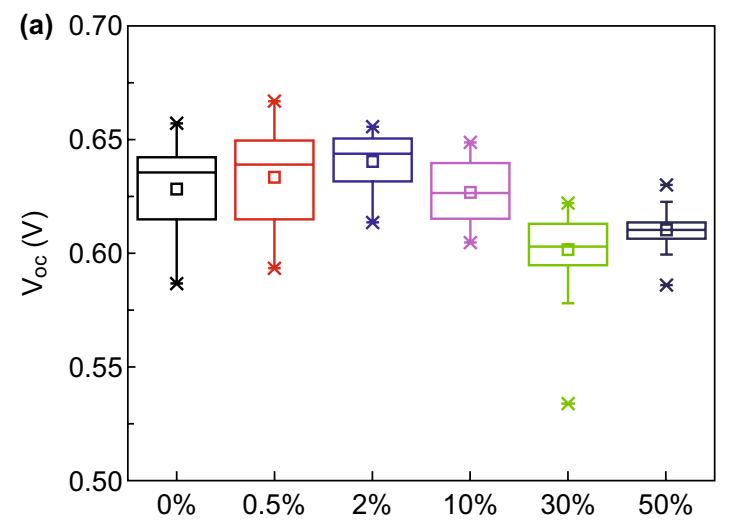

(c)

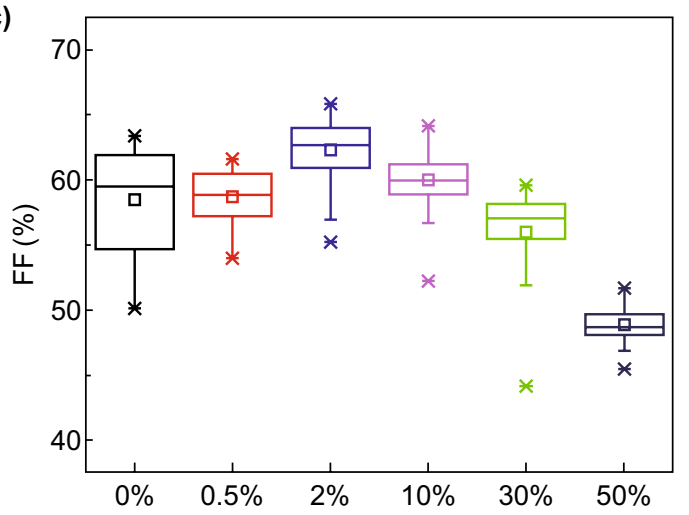

mole ratio to $\mathrm{PbI}_{2}$ is $2 \%$, which achieve the maximum efficiency $(10.78 \%)$ and the maximum $J_{\mathrm{sc}}\left(27.86 \mathrm{~mA} \mathrm{~cm}{ }^{-2}\right)$ (average $J_{\mathrm{sc}}$ is $\sim 25.88 \mathrm{~mA} \mathrm{~cm}^{-2}$ ) with the absorber thickness of $\sim 420 \mathrm{~nm}$. With the same thickness, the w/o HI device only obtain maximum $J_{\mathrm{sc}}$ of $23.65 \mathrm{~mA} \mathrm{~cm}^{-2}$ and average $J_{\mathrm{sc}}$ of $22.74 \mathrm{~mA} \mathrm{~cm}^{-2}$, indicating the carriers diffusion length of w/o HI devices is shorter than w/HI ones.

The current density-voltage $(J-V)$ characteristics of optimal w/HI and w/o HI devices are shown in Fig. $5 b$ and the device parameters are summarized in inset table. The optimal w/HI-PbS solar cell reaches a PCE of $10.78 \%$ (average value $\sim 10.37 \%$ ) with a $V_{\text {oc }}$ of $0.65 \mathrm{~V}$, a $J_{\text {sc }}$ of $25.26 \mathrm{~mA} \mathrm{~cm}^{-2}$, and an FF of 0.66 , which is superior to the w/o-based device with an optimal PCE of $9.56 \%\left(V_{\text {oc }}=0.62 \mathrm{~V}\right.$, $J_{\mathrm{sc}}=24.48 \mathrm{~mA} \mathrm{~cm}^{-2}$, and $\left.\mathrm{FF}=0.63\right)$. The EQE of both devices are shown in Fig. 5c. At the 930-940 nm exciton peaks, w/HI devices reach $65 \%$. On contrary, the control device value is just close to $53 \%$. We consider this improvement related to suppression of the hydroxide species and enhancement of iodide passivation for w/HI films. The w/
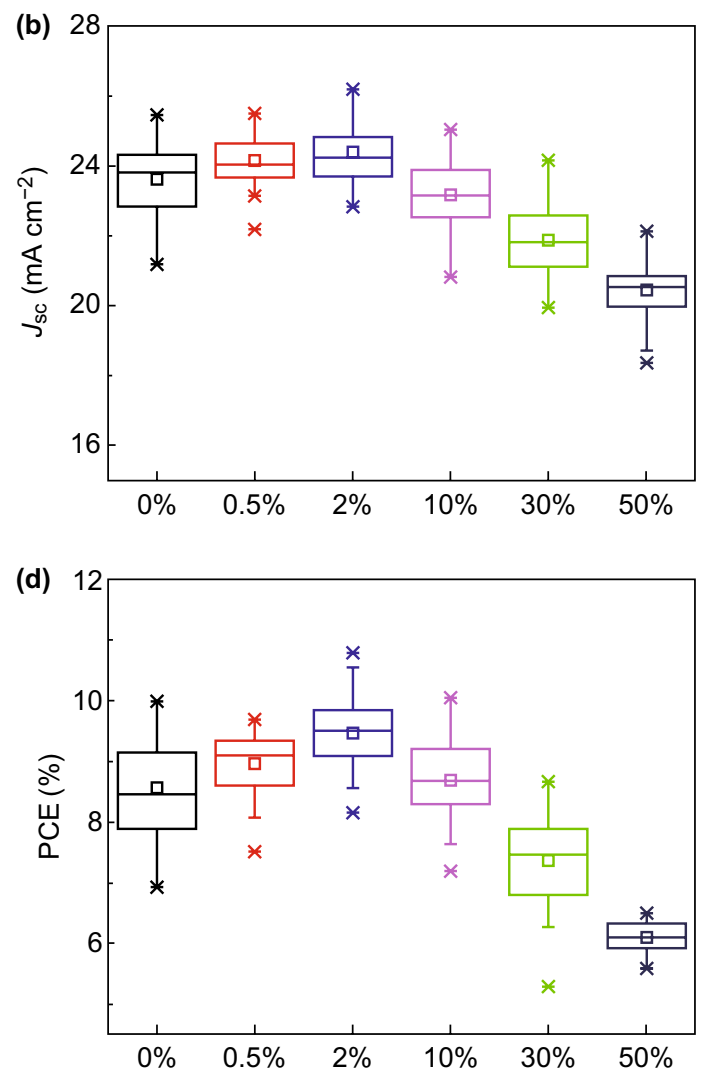

Fig. 4 Device performance parameters of $\mathbf{a} V_{\mathrm{oc}}, \mathbf{b} J_{\mathrm{sc}}, \mathbf{c} \mathrm{FF}$, and $\mathbf{d}$ PCE distribution box with different mole ratio of $\mathrm{HI}$ acid added in $\mathrm{PbI}{ }_{2}-\mathrm{PbS}$ solution 
(a)

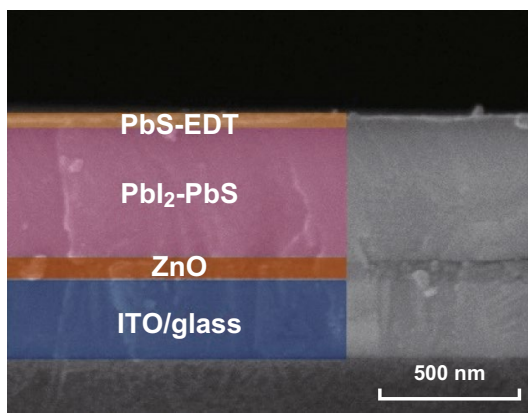

(c)

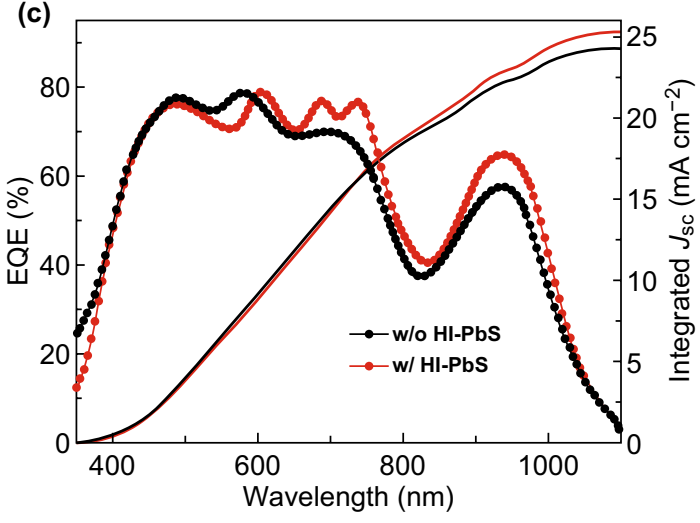

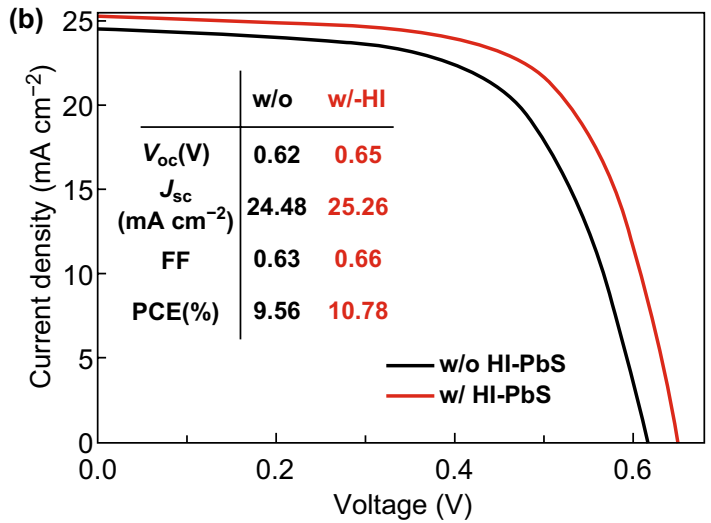

(d)

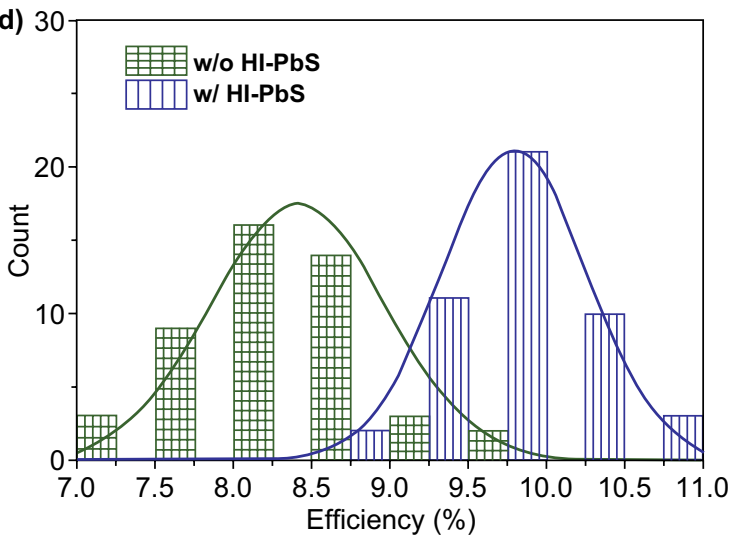

Fig. 5 Device architecture and performances. a The PbS-QDs solar cell device structure. b Representative $J-V$ characteristics of w/o and w/HI devices under simulated AM $1.5 \mathrm{G}$ illumination $\left(100 \mathrm{~mW} \mathrm{~cm}^{-2}\right)$. c EQE spectra and integrated $J_{\mathrm{sc}}$ of w/and w/o HI devices. d Statistic histograms of PCE for above two kinds of devices

HI device shows narrower efficiency distribution, and their average efficiency is much higher than control devices. The statistic histograms of PCE for w/and w/o HI treated PbSQDs solar cells are summarized in Fig. 5d. Device physical characterizations were further utilized to unfold the background mechanism.

According to the $J-V$ curve, the performance enhancement of w/HI PbS-QDs-based devices mainly came from the improvement in $V_{\text {oc }}$ and FF. In order to gain insight into the physical origins of the improvement performance, we firstly measured the temperature-dependent $J-V$ characteristics for generation-recombination in these devices. By extrapolating the $V_{\text {oc }}$ to $T=0 \mathrm{~K}$ (Fig. 6a), the activation energy [32, 33] of w/and w/o HI devices are 1.07 and $1.02 \mathrm{eV}$, respectively. Both of them are less than the bandgap of $\mathrm{PbS}$-QDs $\left(E_{\mathrm{g}} \sim 1.36 \mathrm{eV}\right)$, indicating obvious existence of interfacial recombination in these devices. And the activation energy value of w/HI device is slightly higher than that of the w/o HI devices, which is ascribed to the better passivation in the interface of $\mathrm{ZnO}$ and QDs. To investigate the recombination mechanisms of above two kinds of devices, the light-intensity dependences of $J_{\text {sc }}$ and $V_{\text {oc }}$ were measured to get the device ideality factor $\mathrm{n}[9,32]$. As shown in Fig. 6b, c, the slope of the plot for w/HI device is much lower $(1.23 \mathrm{kT} / \mathrm{q})$ than that of control device $(1.59 \mathrm{kT} / \mathrm{q})$, which indicate $\mathrm{w} / \mathrm{HI}$ devices greatly reduced the trap-assisted recombination [22, 32]. Hence, it can help to improve $V_{\mathrm{oc}}$ and FF.

We further characterized charge-transfer and recombination kinetics in above two kinds of solar cells. We used transient photovoltage decay under the open-circuit condition to characterize solar cells. The extracted charge-recombination lifetime $\left(\tau_{\mathrm{r}}\right)$ of $\mathrm{w} / \mathrm{HI}$ devices is substantially longer than that of control device (106 versus $73.8 \mu$ s) (Fig. 6d). In Fig. 6e, the obtained charge-transport time of w/HI devices $\left(\tau_{\mathrm{t}} \sim 2.82 \mu \mathrm{s}\right)$ is shorter than the value of control one $\left(\tau_{\mathrm{t}} \sim 4.23 \mu \mathrm{s}\right)$, indicating the faster photocarrier transport in w/HI devices. From $V_{\mathrm{oc}}$ and $J_{\mathrm{sc}}$ transient decay dynamics, it concluded that both 

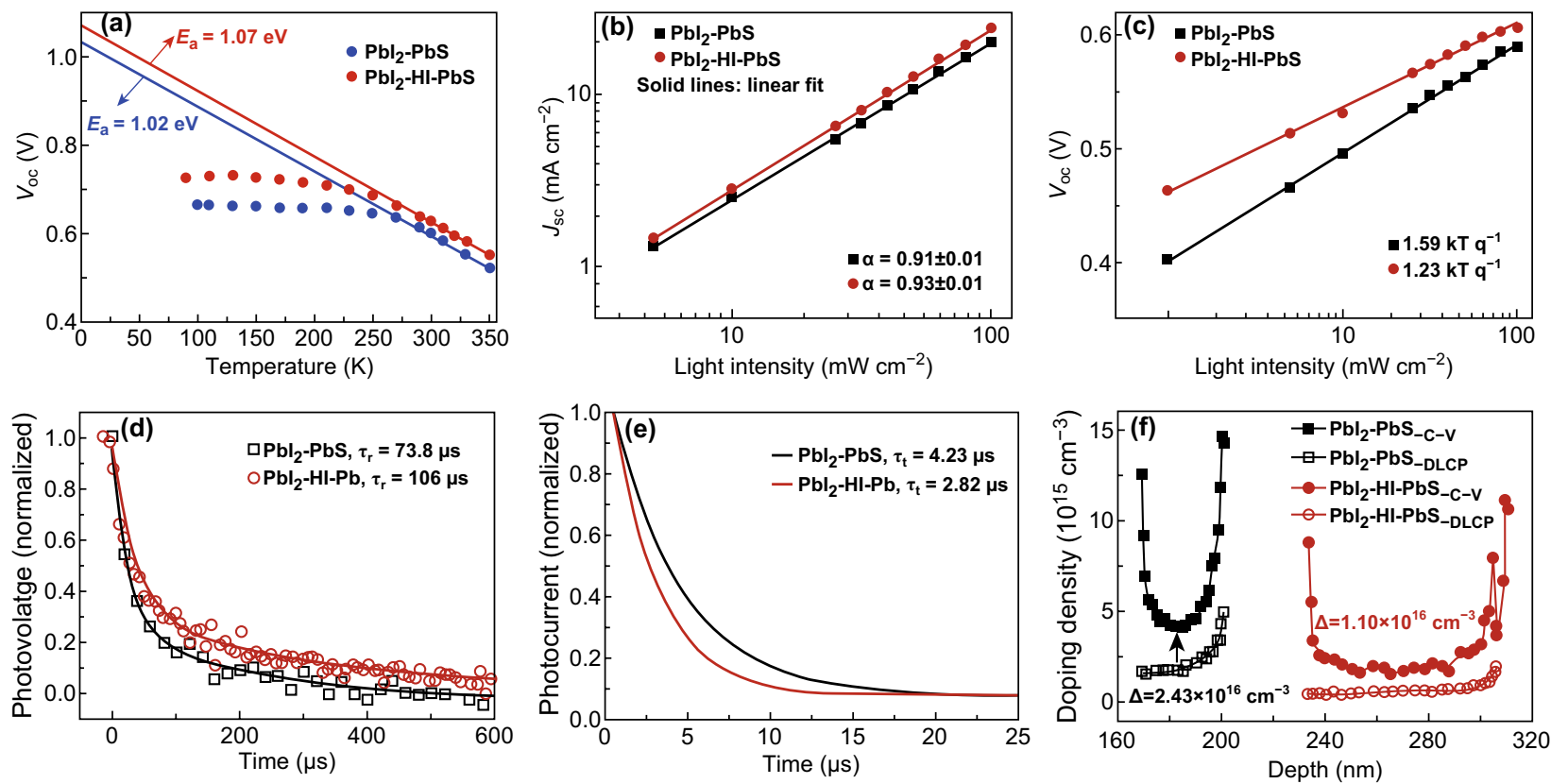

Fig. 6 Physical characterizations of w/o and w/HI devices. a Temperature dependence of $V_{\text {oc }}$ under $100 \mathrm{~mW} \mathrm{~cm}^{-2}$ light intensity. Solid line: linear fits. b Light-intensity dependence of $J_{\mathrm{sc}}$. Solid lines: linear fits. c Light-intensity dependence of $V_{\mathrm{oc}}$. Solid lines: logarithmic fits. d Charge recombination and e transport lifetime calculated by TPV and TPC, respectively. f Interfacial defects analysis from $C-V$ and DLCP results

interfacial and bulk qualities of w/HI devices were improved compared with control devices.

Based on above analyses, it demonstrated that $\mathrm{HI}$ treated $\mathrm{PbS}$-QDs suppressed the defects at $\mathrm{ZnO} / \mathrm{PbS}-\mathrm{QDs}$ heterojunction interface and QDs bulk layers. Capacitance-voltage $(C-V)$ profiling and deep-level capacitance profiling (DLCP) measurements were carried out on these devices to investigate the related defect information. In general, $C-V$ content includes responses from free carriers [34], defects in the bulk, and interfacial defects, while DLCP result is only sensitive to free carriers and bulk defects $[33,35]$. Thus, the difference between $N_{C-V}$ (defect density calculated from $C-V$ measurement) and $N_{\text {DLCP }}$ (defect density calculated from DLCP measurement) reflects defect density [33] at the $\mathrm{ZnO} /$ $\mathrm{PbS}$ interface. As shown in Fig. 6f, the above two devices based DLCP curves are not overlapped and the value of w/ $\mathrm{HI}$ device is lower than w/o $\mathrm{HI}$ one. Combining $C-V$ and DLCP measurements, we calculated the interfacial defect density as $4.07 \times 10^{10} \mathrm{~cm}^{-2}$ in $\mathrm{w} / \mathrm{HI}$ treated one, which was near three times lower than that in w/o HI treated device $\left(1.35 \times 10^{11} \mathrm{~cm}^{-2}\right)$. In order to obtain the QD surface defect density of bulk PbS films, we carried out the DLCP measurement at low $(150 \mathrm{~K})$ and room temperature $(300 \mathrm{~K})$ (Fig.
S10); the difference of doping density is bulk trap states [35, 36]. The extracted surface defect concentration of bulk $\mathrm{PbS}$ films from $\mathrm{w} / \mathrm{HI}$ device is $\sim 1.5 \times 10^{15} \mathrm{~cm}^{-3}$, which is threefold lower than w/o HI PbS-QDs device $\left(\sim 5 \times 10^{15} \mathrm{~cm}^{-3}\right)$. These results are in accordance with the results of TPV (Fig. 6d) and SCLC analysis (Fig. S11).

To confirm the above physical results and deduce the energy band structure, we built a one-dimension model of optoelectronic device (Fig. 7a) that took into account the electron affinity of CQDs with w/and w/o HI treatments, absorption profiles, trap density, and carrier mobility referring from our previously work $[37,38]$ or reported values from iodide-passivated films [8] (Fig. S12 and Table S2). From these simulation results (Fig. 7b), here is showed similar results with real w/o and w/HI devices. That is, the lower trap density of device would get the better performance. From the simulation results, HI additive could enhance the performances ( $V_{\mathrm{oc}}, J_{\mathrm{sc}}$, and FF) for PbS-QDs devices by suppressing the detrimental effect of hydroxyl ligands. Based on the above conclusions, a schematic illustration of proposed photocarrier transport and transfer for w/o and w/HI devices were plotted as shown in Fig. 7c. For w/o HI-based devices, the sub-bandgap states induced by hydroxyl ligand would 

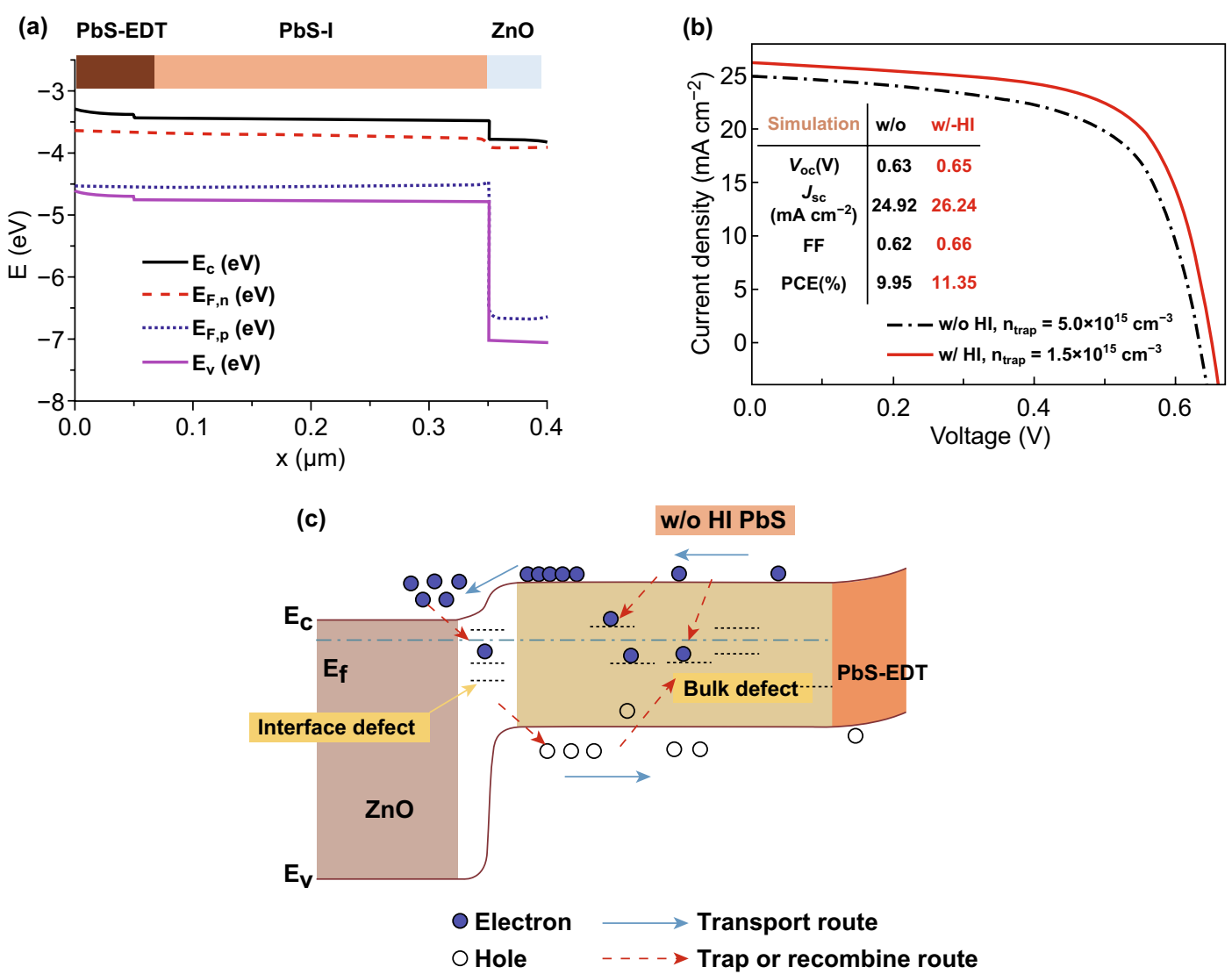

Fig. 7 a The energy band from SCAPS modeling for PbS-QDs solar cell. b Current-voltage characteristics under AM 1.5G illumination for w/and w/o HI devices. c Schematic illustration of proposed photocarrier transport and transfer for w/o and w/HI devices based on simulation and device physics results

trap the carriers and promote non-radiative recombination, leading to lower $J_{\text {sc }}$ and $V_{\text {oc }}$. Those results confirmed the importance of removing hydroxyl and its detrimental effect in PbS-QDs for achieving high efficiency photovoltaics.

As $\mathrm{HI}$-processed $\mathrm{PbI}_{2}-\mathrm{PbS}$ solar cells suppressed hydroxyls and converted them with iodine passivation, which was expected to hold better stability. Thus, we measured the device ambient storing stability at room temperature. As shown in Fig. S13a, w/HI devices could keep $60 \%$ of their original PCE after 1500 min under illumination, which were much better than w/o devices ( 40\% after $1500 \mathrm{~min})$. The main performance decays were from the loss of $J_{\mathrm{sc}}$ and FF (Fig. S13b-d), which may be degraded by oxidation effects [2, 11]. For higher HI concentration (50\%)-processed-PbS solar cells, they decayed more seriously to $50 \%$ of their initial PCE only after 60 min illumination. This fast degradation was mainly caused by overtreatment by HI, leading to the fusion or etching in PbS-QDs, which led to be more sensitive to ambient atmosphere. It also indicated that the amount of additive HI needs to be precise controlled.

Compared with previous work by Jo et al. [31], the motivation and key finding of them are different to ours. They mostly focused on large-diameter (4-6 nm, $E_{\mathrm{g}}<1.1 \mathrm{eV}$ ) CQDs for harvest energy in the infrared region of the solar spectrum. They employed the hydroiodic acid in order to increase the chemical reactivity to facilitate high CQD packing and passivation for large size infrared CQDs (IRQDs). Our work focused on the improved performance for wider-bandgap CQDs whose bandgaps were within the range of 1.3-1.5 eV, closed to the idea band gap for single junction solar cell. In our case, it is not the key problem to remove the oleate ligand after solution-processed ligandexchange method referring from our FT-IR spectra. And the wider-bandgap CQDs of ours used for single junction solar cells focused on the elimination for sub-bandgap states and improvement in passivation. Therefore, we paid more 
attention about device physics characterization to know about the mechanism of hydroxyl ligand to PbS-QDs solar cell performance. Meanwhile, we extracted the optimal recipe for hydroxyl removal treatment. In general, the reported work [31] and ours focused on different motivations and different size QD for harvesting different region of the solar spectrum. And these two works can hold the complementary role for each other.

\section{Conclusions}

In summary, we demonstrated a halide ligand additive strategy to suppress hydroxyl ligands and convert them with iodine ligand passivation. The approach allowed the halide acid, HI, to access target $\mathrm{Pb}-\mathrm{OH}$ bonding based on a deprotonation reaction between $\mathrm{HI}$ additive and hydroxyl ligands. Utilizing optimal concentration HI treatment, QDs could obtain higher $\mathrm{I} / \mathrm{Pb}$ ratio $(0.75: 1)$ with improved passivation on QDs surface. Such treatment strategy promised thicker absorber layer with higher mobility of carriers in solar cells, delivering a PCE of $10.78 \%$. Systematic physical analyses to the improvement mechanism were originated from the suppression of interfacial and bulk defect density. Thus, the device $V_{\text {oc }}$ and FF could be significantly improved as well as the device stability. The present ingenious strategy was expected to continuously promote PbS-QDs ink technology, and also be applicable for other CQD-based optoelectronic devices.

Acknowledgements This work was financially supported by National Natural Science Foundation of China (61874165, 51761145048, and 21833009), the Foundation of Shenzhen Science and Technology Innovation Committee (JCYJ20170413113645633) and Major State Basic Research Development Program of China (2016YFB0700702). C. Cheng also thanks the Guangdong-Hong Kong joint innovation project (2016A050503012), and Guangdong Natural Science Funds for Distinguished Young Scholars (2015A030306044). The authors thank Wuhan Institute of Physics and Mathematics, Chinese Academy of Sciences (CAS) for Solid-state NMR measurements, Material Science and Engineering of HUST, Testing Center of HUST, the Center for Nanoscale Characterization \& Devices (CNCD), WNLO-HUST for facility access.

Open Access This article is licensed under a Creative Commons Attribution 4.0 International License, which permits use, sharing, adaptation, distribution and reproduction in any medium or format, as long as you give appropriate credit to the original author(s) and the source, provide a link to the Creative Commons licence, and indicate if changes were made. The images or other third party material in this article are included in the article's Creative
Commons licence, unless indicated otherwise in a credit line to the material. If material is not included in the article's Creative Commons licence and your intended use is not permitted by statutory regulation or exceeds the permitted use, you will need to obtain permission directly from the copyright holder. To view a copy of this licence, visit http://creativecommons.org/licenses/by/4.0/.

Electronic supplementary material The online version of this article (https://doi.org/10.1007/s40820-020-0372-z) contains supplementary material, which is available to authorized users.

\section{References}

1. F.P. García de Arquer, A. Armin, P. Meredith, E.H. Sargent, Solution-processed semiconductors for next-generation photodetectors. Nat. Rev. Mater. 2, 16100 (2017). https://doi. org/10.1038/natrevmats.2016.100

2. J. Tang, K.W. Kemp, S. Hoogland, K.S. Jeong, H. Liu et al., Colloidal-quantum-dot photovoltaics using atomic-ligand passivation. Nat. Mater. 10(10), 765-771 (2011). https://doi. org/10.1038/nmat3118

3. C.H. Chuang, P.R. Brown, V. Bulovic, M.G. Bawendi, Improved performance and stability in quantum dot solar cells through band alignment engineering. Nat. Mater. 13(8), 796-801 (2014). https://doi.org/10.1038/nmat3984

4. D.M. Kroupa, M. Voros, N.P. Brawand, B.W. McNichols, E.M. Miller et al., Tuning colloidal quantum dot band edge positions through solution-phase surface chemistry modification. Nat. Commun. 8, 15257 (2017). https://doi.org/10.1038/ncomm s15257

5. N. Zhang, D.C. Neo, Y. Tazawa, X. Li, H.E. Assender, R.G. Compton, A.A. Watt, Narrow band gap lead sulfide hole transport layers for quantum dot photovoltaics. ACS Appl. Mater. Interfaces 8(33), 21417-21422 (2016). https://doi. org/10.1021/acsami.6b01018

6. J.W. Jo, Y. Kim, J. Choi, F.P.G. de Arquer, G. Walters et al., Enhanced open-circuit voltage in colloidal quantum dot photovoltaics via reactivity-controlled solution-phase ligand exchange. Adv. Mater. 29(43), 1703627 (2017). https://doi. org/10.1002/adma.201703627

7. M. Yuan, D. Zhitomirsky, V. Adinolfi, O. Voznyy, K.W. Kemp et al., Doping control via molecularly engineered surface ligand coordination. Adv. Mater. 25(39), 5586-5592 (2013). https://doi.org/10.1002/adma201302802

8. X. Lan, O. Voznyy, A. Kiani, F.P. Garcia de Arquer, A.S. Abbas et al., Passivation using molecular halides increases quantum dot solar cell performance. Adv. Mater. 28(2), 299304 (2016). https://doi.org/10.1002/adma.201503657

9. D. Mandal, P.N. Goswami, A.K. Rath, Thiol, halometallate, mutually passivated quantum dot ink for photovoltaic application. ACS Appl. Mater. Interfaces 11(29), 26100-26108 (2019). https://doi.org/10.1021/acsami.9b07605

10. A.R. Kirmani, F.P. García de Arquer, J.Z. Fan, J.I. Khan, G. Walters et al., Molecular doping of the hole-transporting layer 
for efficient, single-step-deposited colloidal quantum dot photovoltaics. ACS Energy Lett. 2(9), 1952-1959 (2017). https:// doi.org/10.1021/acsenergylett.7b00540

11. Z. Ning, O. Voznyy, J. Pan, S. Hoogland, V. Adinolfi et al., Air-stable n-type colloidal quantum dot solids. Nat. Mater. 13(8), 822-828 (2014). https://doi.org/10.1038/nmat4007

12. M. Liu, F.P. de Arquer, Y. Li, X. Lan, G.H. Kim et al., Doublesided junctions enable high-performance colloidal-quantumdot photovoltaics. Adv. Mater. 28(21), 4142-4148 (2016). https://doi.org/10.1002/adma.201506213

13. D.A. Barkhouse, R. Debnath, I.J. Kramer, D. Zhitomirsky, A.G. Pattantyus-Abraham et al., Depleted bulk heterojunction colloidal quantum dot photovoltaics. Adv. Mater. 23(28), 3134-3138 (2011). https://doi.org/10.1002/adma.201101065

14. L. Hu, Z. Zhang, R.J. Patterson, Y. Hu, W. Chen et al., Achieving high-performance PbS quantum dot solar cells by improving hole extraction through Ag doping. Nano Energy 46, 212 219 (2018). https://doi.org/10.1016/j.nanoen.2018.01.047

15. M. Liu, Y. Chen, C.S. Tan, R. Quintero-Bermudez, A.H. Proppe et al., Lattice anchoring stabilizes solution-processed semiconductors. Nature 570, 96-101 (2019). https://doi. org/10.1038/s41586-019-1239-7

16. H. Aqoma, M. Al Mubarok, W.T. Hadmojo, E.H. Lee, T.W. Kim et al., High-efficiency photovoltaic devices using trapcontrolled quantum-dot ink prepared via phase-transfer exchange. Adv. Mater. 29(19), 1605756 (2017). https://doi. org/10.1002/adma.201605756

17. M. Liu, O. Voznyy, R. Sabatini, F.P. García de Arquer, R. Munir et al., Hybrid organic-inorganic inks flatten the energy landscape in colloidal quantum dot solids. Nat. Mater. 16, 258 (2016). https://doi.org/10.1038/nmat4800

18. D. Zherebetskyy, M. Scheele, Y. Zhang, N. Bronstein, C. Thompson et al., Hydroxylation of the surface of $\mathrm{PbS}$ nanocrystals passivated with oleic acid. Science 344(6190), 1380-1384 (2014). https://doi.org/10.1126/science.1252727

19. Z. Ning, H. Dong, Q. Zhang, O. Voznyy, E.H. Sargent, Solar cells based on inks of n-type colloidal quantum dots. ACS Nano 8(10), 10321-10327 (2014). https://doi.org/10.1021/ nn503569p

20. P.R. Brown, R.R. Lunt, N. Zhao, T.P. Osedach, D.D. Wanger, L.Y. Chang, M.G. Bawendi, V. Bulovic, Improved current extraction from $\mathrm{ZnO} / \mathrm{PbS}$ quantum dot heterojunction photovoltaics using a $\mathrm{MoO}_{3}$ interfacial layer. Nano Lett. 11(7), 2955-2961 (2011). https://doi.org/10.1021/nl201472u

21. Y. Cao, A. Stavrinadis, T. Lasanta, D. So, G. Konstantatos, The role of surface passivation for efficient and photostable PbS quantum dot solar cells. Nat. Energy 1(4), 16035 (2016). https://doi.org/10.1038/nenergy.2016.35

22. Y. Wang, K. Lu, L. Han, Z. Liu, G. Shi et al., In situ passivation for efficient $\mathrm{PbS}$ quantum dot solar cells by precursor engineering. Adv. Mater. 30(16), e1704871 (2018). https:// doi.org/10.1002/adma.201704871

23. D.K. Ko, A. Maurano, S.K. Suh, D. Kim, G.W. Hwang et al., Photovoltaic performance of $\mathrm{PbS}$ quantum dots treated with metal salts. ACS Nano 10(3), 3382-3388 (2016). https://doi. org/10.1021/acsnano.5b07186
24. A.H. Ip, S.M. Thon, S. Hoogland, O. Voznyy, D. Zhitomirsky et al., Hybrid passivated colloidal quantum dot solids. Nat. Nanotechnol. 7(9), 577-582 (2012). https://doi.org/10.1038/ nnano.2012.127

25. X. Lan, O. Voznyy, F.P. Garcia de Arquer, M. Liu, J. Xu et al., $10.6 \%$ certified colloidal quantum dot solar cells via solventpolarity-engineered halide passivation. Nano Lett. 16(7), 4630-4634 (2016). https://doi.org/10.1021/acs.nanolett.6b019 57

26. J. Chen, Q. Ma, X.J. Wu, L. Li, J. Liu, H. Zhang, Wet-chemical synthesis and applications of semiconductor nanomaterialbased epitaxial heterostructures. Nano-Micro Lett. 11, 86 (2019). https://doi.org/10.1007/s40820-019-0317-6

27. Y. Bai, Y. Lu, K. Wang, Z. Cheng, Y. Qu et al., Rapid isolation and multiplexed detection of exosome tumor markers via queued beads combined with quantum dots in a microarray. Nano-Micro Lett. 11, 59 (2019). https://doi.org/10.1007/s4082 0-019-0285-X

28. M. Li, Y. Gu, Y. Liu, Y. Li, Z. Zhang, Interfacial improvement of carbon fiber/epoxy composites using a simple process for depositing commercially functionalized carbon nanotubes on the fibers. Carbon 52, 109-121 (2013). https://doi. org/10.1016/j.carbon.2012.09.011

29. A. Stavrinadis, S. Pradhan, P. Papagiorgis, G. Itskos, G. Konstantatos, Suppressing deep traps in $\mathrm{PbS}$ colloidal quantum dots via facile iodide substitutional doping for solar cells with efficiency $>10 \%$. ACS Energy Lett. 2(4), 739-744 (2017). https://doi.org/10.1021/acsenergylett.7b00091

30. S. Chen, T.W. Goh, D. Sabba, J. Chua, N. Mathews, C. Huan, T.C. Sum, Energy level alignment at the methylammonium lead iodide/copper phthalocyanine interface. Appl. Mater. 2(8), 081512 (2014). https://doi.org/10.1063/1.4889844

31. J.W. Jo, J. Choi, F.P. Garcia de Arquer, A. Seifitokaldani, B. Sun, Y. Kim et al., Acid-assisted ligand exchange enhances coupling in colloidal quantum dot solids. Nano Lett. 18(7), 4417-4423 (2018). https://doi.org/10.1021/acs.nanolett.8b014 70

32. C.H. Chuang, A. Maurano, R.E. Brandt, G.W. Hwang, J. Jean, T. Buonassisi, V. Bulovic, M.G. Bawendi, Open-circuit voltage deficit, radiative sub-bandgap states, and prospects in quantum dot solar cells. Nano Lett. 15(5), 3286-3294 (2015). https://doi.org/10.1021/acs.nanolett.5b00513

33. L. Wang, D.-B. Li, K. Li, C. Chen, H.-X. Deng et al., Stable $6 \%$-efficient $\mathrm{Sb}_{2} \mathrm{Se}_{3}$ solar cells with a $\mathrm{ZnO}$ buffer layer. Nat. Energy 2(4), 17046 (2017). https://doi.org/10.1038/nener gy.2017.46

34. C. Chen, D.C. Bobela, Y. Yang, S. Lu, K. Zeng et al., Characterization of basic physical properties of $\mathrm{Sb}_{2} \mathrm{Se}_{3}$ and its relevance for photovoltaics. Front. Optoelectron. 10(1), 18-30 (2017). https://doi.org/10.1007/s12200-017-0702-z

35. J.T. Heath, J.D. Cohen, W.N. Shafarman, Bulk and metastable defects in $\mathrm{CuIn}_{1-\mathrm{x}} \mathrm{GaxSe}_{2}$ thin films using drive-level capacitance profiling. J. Appl. Phys. 95(3), 1000-1010 (2004). https ://doi.org/10.1063/1.1633982

36. D. Hsin-Sheng, Y. Wenbing, B. Brion, H. Chia-Jung, L. Bao, Y. Yang, The role of sulfur in solution-processed 
$\mathrm{Cu}_{2} \mathrm{ZnSn}(\mathrm{S}, \mathrm{Se})_{4}$ and its effect on defect properties. Adv. Funct. Mater. 23(11), 1466-1471 (2013). https://doi.org/10.1002/ adfm.201201732

37. J. Khan, X. Yang, K. Qiao, H. Deng, J. Zhang et al., Lowtemperature-processed $\mathrm{SnO}_{2}-\mathrm{Cl}$ for efficient $\mathrm{PbS}$ quantum-dot solar cells via defect passivation. J. Mater. Chem. A 5(33), 17240-17247 (2017). https://doi.org/10.1039/C7TA05366E
38. X. Yang, L. Hu, H. Deng, K. Qiao, C. Hu et al., Improving the performance of $\mathrm{PbS}$ quantum dot solar cells by optimizing $\mathrm{ZnO}$ window layer. Nano-Micro Lett. 9(2), 24 (2017). https:// doi.org/10.1007/s40820-016-0124-2 